\title{
Factors influencing particle agglomeration during solid-state sintering
}

\author{
Chao Wang • Shao-Hua Chen
}

Received: 30 May 2011 / Revised: 30 November 2011 / Accepted: 19 December 2011

(C)The Chinese Society of Theoretical and Applied Mechanics and Springer-Verlag Berlin Heidelberg 2012

\begin{abstract}
Discrete element method (DEM) is used to study the factors affecting agglomeration in three-dimensional copper particle systems during solid-state sintering. A new parameter is proposed to characterize agglomeration. The effects of a series of factors are studied, including particle size, size distribution, inter-particle tangential viscosity, temperature, initial density and initial distribution of particles on agglomeration. We find that the systems with smaller particles, broader particle size distribution, smaller viscosity, higher sintering temperature and smaller initial density have stronger particle agglomeration and different distributions of particles induce different agglomerations. This study should be very useful for understanding the phenomenon of agglomeration and the micro-structural evolution during sintering and guiding sintering routes to avoid detrimental agglomeration.
\end{abstract}

Keywords Solid-state sintering - Discrete element simulation $\cdot$ Agglomeration · Densification

\section{Introduction}

Agglomeration is a result of drastic rearrangement of particles at the early stage of sintering, during which shrinkage occurs within many regions and the particles in each region are drawn inwards towards the geometrical center of the re-

The project was supported by the National Natural Science Foundation of China (10972220, 11125211 and 11021262) and 973 Project (2012CB937500).

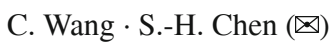

State Key Laboratory of Nonlinear Mechanics,

Institute of Mechanics,

Chinese Academy of Sciences,

100190 Beijing, China

e-mail: chenshaohua72@hotmail.com gion. Thus, agglomerates gradually take place accompanying with the increase of porosity between them [1], which would lead to retardation of the general shrinkage. Agglomeration is widely observed in metal and ceramic solid-state sintering, especially in nano-particle sintering. Petzow et al. [2] observed agglomeration during sintering of planar arrays of uniform copper spheres at $950{ }^{\circ} \mathrm{C}$. Similar phenomena have been found in irregular arrays of glass spheres [3] and copper particles [4], a compact of fine tungsten powder [5], fine chromium oxide powder compacts [5] and alumina powder compacts [6]. Kuo et al. [7] found that almost all the powder of alumina- 8 vol\% zirconia was agglomerated to various sizes from less $1 \mu \mathrm{m}$ to more than $10 \mu \mathrm{m}$ after calcination at $550{ }^{\circ} \mathrm{C}$ for 2 hours. Palmero et al. [8] found a heterogeneous microstructure consisting of agglomerates of ultrafine alumina particles of about 150-200 nm, which was sintered at $1135^{\circ} \mathrm{C}$. Agglomeration was also observed in liquid-phase sintering [9] and in the process of producing fine-sized ceramic powders $[10,11]$.

Agglomeration has shown significant influences on sintering process and compaction in experiments $[12,13]$ and simulations [14-17]. Ciftcioglu et al. [18] investigated the effect of agglomerate strength on sintered density experimentally and achieved a conclusion that the sintered density of yttria powders decreased with increasing agglomerate strength. Rhodes [12] found that agglomerates limited attainable green density, interfered with the development of microstructure, impeded initial-stage sintering kinetics, and limited the potential benefit of fine crystallites in final-stage sintering. Lange et al. [13] found that dry powder routes to powder consolidation could produce large agglomerates, which resulted in large crack-like voids due to differential sintering. Martin et al. [16] studied the morphology and strength of agglomerates using computational simulation and found that the morphology of agglomerates had an obvious effect on the tap density but their strength was a predominant factor affecting green density. Kim et al. [17] considered the rearrangement of agglomerates and found that compacts 
with rearranged agglomerates had higher packing densities than those without rearrangement.

Though the pioneering works disclose some effects of agglomeration on sintering and compaction, some fundamental problems are still open. What factors influence agglomeration and how? How can we describe agglomeration in a quantitative way?

It is a much challenging job to establish theoretical models to study the formation and effects of agglomerates at the present stage. It is because agglomeration during sintering may be influenced by many factors, such as the properties of materials, particle size, size distribution, temperature, and so on. Furthermore, the strength, shape, size and distribution of agglomerates also affect the evolution of microstructure and the final properties of materials. Experimentally, the instantaneous observation of agglomeration in $3 \mathrm{D}$ compacts is so complex and difficult, that the effects of agglomerates are investigated by introducing them into the system before sintering. In fact, agglomerates are always forming during the sintering process. Thanks to the new technology of in situ Xray micro-tomography, which has been used to observe the rearrangement of particles during sintering [19-23], we believe that it will be adopted to study the agglomeration in 3D compacts in the near future.

An alternative approach is numerical simulation. Due to its natural treatment at a particle length scale, recent discrete element method (DEM) [24-27] has shown its capability to study the effect of rearrangement not only in macroscopic aspects, such as densification rates [24, 26, 28], viscosities [26, 29], anisotropic sintering [30], constrained sintering [31], but also in microscopic aspect of crack evolution during sintering $[26,32]$. This method has also shed some lights on our understanding of certain problems about agglomerates. Kim et al. [17] investigated the effect of agglomerate rearrangement on packing density. Martin et al. [16] studied the effect of morphology and strength of agglomerates on packing density. As far as we know, Kadusnikov et al. $[14,15]$ are the first ones to simulate agglomeration ("zonal segregation" used in their papers) in sintering by a sphere-polyhedron model. They find that regular close packing of identical particles does not produce agglomeration while for any irregular systems, e.g. random close packing with pores or with particle size distributions, agglomeration is certain to happen. They further added that the two-particle coalescence rate is another major factor affecting agglomeration. For a particle system, there is a critical two-particle coalescence rate, below which the geometrical irregularities relax during sintering, while above which fluctuations due to imperfections accumulate and agglomeration occurs. They studied the effect of the two-particle coalescence rate on microstructural evolution. However, the two-particle coalescence rate is determined by a series of physicochemical factors including particle size, temperature, viscosity, diffusion coefficient and so on. As a preliminary work, effects of those factors are not discussed in their works.
In this paper, the discrete element method (DEM) similar to the original work done by Cundall [33] will be used, which has been proved to be more appropriate for modeling particle rearrangement. A new parameter named "the variance of distances between neighboring particles" is proposed to characterize the agglomeration. Then the method is used to study effects of particle size, size distribution, temperature, inter-particle tangential viscosity, the initial distribution of particles and the initial volume fraction on the agglomeration in a $3 \mathrm{D}$ copper particle sintering system.

\section{Numerical simulation method}

\subsection{Discrete element method (DEM)}

DEM is used to simulate the sintering process, in which the quantities describing a particle consist of a position vector $\boldsymbol{x}_{i}$, velocity $\dot{\boldsymbol{x}}_{i}$, angular velocity $\boldsymbol{\omega}_{i}$, mass $m_{i}$ and moment of inertia $I_{i}$. At each time step $t$, the interacting force and torque between two adjacent contacting particles are $\boldsymbol{F}_{i j}$ and $\boldsymbol{T}_{i j}$, where $\boldsymbol{T}_{i j}=-(1 / 2) \boldsymbol{r}_{i j} \times \boldsymbol{F}_{i j}$ with $\boldsymbol{r}_{i j}=\boldsymbol{x}_{i}-\boldsymbol{x}_{j}$. The acceleration $\ddot{\boldsymbol{x}}_{i}(t)$, angular acceleration $\dot{\omega}_{i}(t+\Delta t)$, velocity $\dot{\boldsymbol{x}}_{i}(t+\Delta t)$, angular velocity $\boldsymbol{\omega}_{i}(t+\Delta t)$ and position $\boldsymbol{x}_{i}(t+\Delta t)$ can be calculated using the Newton's second law and a verlet type algorithm as follows,

$$
\begin{aligned}
& \ddot{\boldsymbol{x}}_{i}(t)=\frac{1}{m_{i}} \sum_{j \neq i} \boldsymbol{F}_{i j}, \\
& \dot{\boldsymbol{\omega}}_{i}(t)=\frac{1}{I_{i}} \sum_{j \neq i} \boldsymbol{T}_{i j}, \\
& \boldsymbol{x}_{i}(t+\Delta t)=\boldsymbol{x}_{i}(t)+\left(\dot{\boldsymbol{x}}_{i}(t)+\frac{1}{2} \ddot{\boldsymbol{x}}_{i}(t) \Delta t\right) \Delta t, \\
& \dot{\boldsymbol{x}}_{i}(t+\Delta t)=\dot{\boldsymbol{x}}_{i}(t)+\frac{1}{2}\left(\ddot{\boldsymbol{x}}_{i}(t)+\ddot{\boldsymbol{x}}_{i}(t+\Delta t)\right) \Delta t, \\
& \omega_{i}(t+\Delta t)=\omega_{i}(t)+\frac{1}{2}\left(\dot{\boldsymbol{\omega}}_{i}(t)+\dot{\boldsymbol{\omega}}_{i}(t+\Delta t)\right) \Delta t,
\end{aligned}
$$

where $\Delta t$ is a small value, in which the particle $i$ is assumed to interact only with its neighbors and can not move over its neighbors. In the numerical simulation, a real mass $m_{i}$ will require the time step $\Delta t$ to be a very small value, which in turn leads to an expensive simulation time. In order to overcome the drawback, a special technique is adopted in this paper $[25,26,34]$, in which the mass of a particle is scaled up by a factor $\beta$, the acceleration and velocity can be reduced by the same order of magnitude without influencing the equilibrium position of each particle. Thus, a relatively large time step can be chosen. However, the factor $\beta$ should not be too large as analyzed by Henrich et al. [26]. Martin et al. [25] used $10^{13}$ for $\beta$ in their simulations. In the present paper, we choose $\beta=10^{12}$ and $\Delta t=1 \mathrm{~s}$. Actually, we also checked simulations with $\beta=10^{13}$ and $\Delta t=1 \mathrm{~s}$ or $\beta=10^{12}$ and $\Delta t=0.5 \mathrm{~s}$, the same results can be achieved as those presented in the following sections. 


\subsection{Interacting force between adjacent particles}

According to Refs. [24, 28, 31,32], the normal force $N_{\mathrm{s}}$ and the tangential force $T_{\mathrm{s}}$ acting at the interface of two contacting particles are

$N_{\mathrm{s}}=\frac{\pi a_{\mathrm{s}}^{4}}{8 \Delta_{\mathrm{b}}} \frac{\mathrm{d} h}{\mathrm{~d} t}-\pi \gamma_{\mathrm{s}}\left[4 R_{\mathrm{p}}\left(1-\cos \frac{\psi}{2}\right)+a_{\mathrm{s}} \sin \frac{\psi}{2}\right]$,

$T_{\mathrm{s}}=-\eta \frac{\pi a_{\mathrm{s}}^{2} R_{\mathrm{p}}^{2}}{8 \Delta_{\mathrm{b}}} \frac{\mathrm{d} u}{\mathrm{~d} t}$

$\Delta_{\mathrm{b}}=\frac{\Omega}{k T} \delta_{\mathrm{b}} D_{\mathrm{b}}$,

where $D_{\mathrm{b}}=D_{0 \mathrm{~b}} \exp \left(-Q_{\mathrm{b}} / R T\right)$ is the diffusion coefficient for vacancy transport in the grain boundary with thickness $\delta_{\mathrm{b}}$ and activation energy $Q_{\mathrm{b}}, \Omega$ is the atomic volume, $k$ is the Boltzmann constant, $T$ is the temperature, $h$ is the indentation depth between two spherical particles, $\gamma_{\mathrm{s}}$ is the surface energy, $R_{\mathrm{p}}$ is the radius of the particle, $\psi$ is the dihedral angle, $a_{\mathrm{s}}$ is the sintering contact radius, which grows according to the Coble's model $a_{\mathrm{s}}=\sqrt{2 h R_{\mathrm{p}}} . \mathrm{d} u / \mathrm{d} t$ is the tangential component of relative velocity at the contact and $\eta$ is the viscous parameter.

Equation (2) consists of grain boundary diffusion and surface diffusion, which is originally and strictly obtained by Bouvard and McMeeking [35] and Parhami and McMeeking [29] from the physics and mechanics view-points. The first term on the right hand side of Eq. (2a) is the normal viscous force resisting the relative motion normal to the contact interface of two adjacent particles, which has also been adopted in Refs. [26, 30, 36, 37]. The second term of Eq. (2a) denotes the sintering force pulling two adjacent particles together. The tangential contact force $T_{\mathrm{s}}$ in Eq. (2b) opposes the tangential component of relative velocity at the contact.

We introduce an equivalent radius $R^{*}=2 R_{1} R_{2} /\left(R_{1}+R_{2}\right)$ to replace $R_{\mathrm{p}}$ in Eqs. (2a) and (2b) for calculating $N_{\mathrm{s}}$ and $T_{\mathrm{s}}$ between two particles of radii $R_{1}$ and $R_{2}$. The treatment has been suggested by Parhami et al. [38] and is in good accord with numerical simulations [39].

\section{Simulation model and parameters}

Three 3D models are used in the present paper. The first one is a cube containing 1185 uniform spheres with a boundary length 20 times of the particle radius corresponding to the initial volume fraction of $62 \%$, which is used to investigate effects of the particle size, temperature, initial particle distribution and tangential viscosity on agglomeration. The second one is a cube containing 1200 non-uniform spheres with the boundary length 20 times of the average particle radius, which will be used to investigate the effect of particle size distribution on agglomeration. Three kinds of standard deviations $0.02,0.06$ and 0.08 for the particle size distributions are considered. The third one is a cube containing 1200 uniform spheres with the boundary length $21.58,21.30,20.78$ and 20.31 times of the particle radius, respectively, which denote different volume fractions 50\%, 52\%, 56\% and $60 \%$ of particles.

The value of the viscous parameter is problematic. Martin et al. [31] argued that the normal viscosity term in Eq. (2a) should be of the same order or larger than the tangential viscosity term in Eq. (2b) when the normal and tangential relative velocity are of the same order. A large $\eta$ requires a small time step to ensure convergence of calculation, which leads to a long CPU time. We find that a large $\eta$ could be used in systems with small particles, e.g. $22 \mu \mathrm{m} . \eta \leq 2$ is set in the present paper.

Usually, sintering process can be divided into three stages, in which grain growth is significant at the final stage of sintering when the relative density of the system is larger than 0.87 [40]. However, it is no longer reasonable to model the sintering body as a packing of spherical particles above this relative density. The relative density of sintering body in the present simulations does not exceed 0.87 and the effect of grain growth is not included [20]. Other physical parameters used in the simulations are listed in Table 1.

Table 1 Parameters used in the discrete element simulation of copper sintering (from Ref. [28])

\begin{tabular}{lllll}
\hline$\delta_{\mathrm{b}} D_{0 \mathrm{~b}} /\left(\mu \mathrm{m}^{3} \cdot \mathrm{s}^{-1}\right)$ & $\gamma_{\mathrm{s}} /\left(\mathrm{J} \cdot \mathrm{m}^{-2}\right)$ & $\Omega / \mathrm{nm}^{3}$ & $Q_{\mathrm{b}} /\left(\mathrm{kJ} \cdot \mathrm{mol}^{-1}\right)$ & $\psi /\left(^{\circ}\right)$ \\
\hline 5120 & 1.72 & 0.0118 & 105 & 146 \\
\hline
\end{tabular}

Figure 1 shows the visualization of a sintering system for the first model with particle size $22 \mu \mathrm{m}$, temperature $1050{ }^{\circ} \mathrm{C}$, and tangential viscosity 0.001 . Each particle is colored by its coordination number. Figure 1a depicts the initial state and Fig. $1 \mathrm{~b}$ the state with densification $\left(D-D_{0}\right) / D_{0}=0.34$, where $D_{0}$ and $D$ are the initial and the current relative densities, respectively.

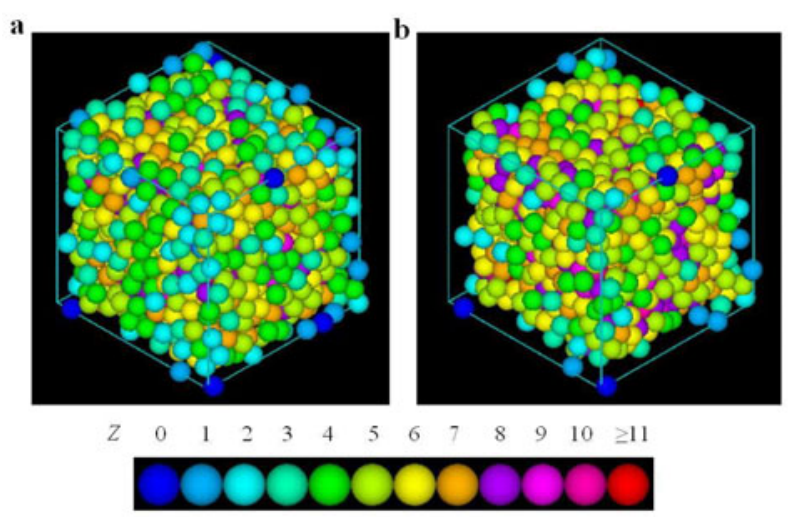

Fig. 1 a Snapshots of the initial state and $\mathbf{b}$ intermediate state with $\left(D-D_{0}\right) / D_{0}=0.34$ for a typically free sintering simulations using DEM. Different color indicates the coordination number of each particle 


\subsection{The "deviation angle" $\theta$}

The "deviation angle" $\theta$ is the one between the displacement vector - going from the initial position of the particle center to its current position at a given sintering time — and the vector from the initial position of the particle center to the center of the whole system. This definition has also been adopted by Olmos et al. [20].

\subsection{Variance of distances between neighboring particles}

A new parameter "variance" is proposed to denote the variance of distances between neighboring particles. This parameter, which characterizes the uniformity of the distribution of particles in the system, can quantitatively measure the degree of agglomeration. The meaning of "degree of particle agglomeration" in this paper is equivalent to that of "degree of uniformity of the distribution of particles".

To calculate this parameter, neighbors of each particle will be detected first at the beginning of simulations and will not change later. A threshold value $r_{\mathrm{c}}$ is introduced to find the neighbors of a particle. A particle $P_{j}$ $\left(P_{j} \neq P_{i}, j=1,2, \cdots, M\right)$ being the neighbor of a particle $P_{i}(i=1,2, \cdots, N)$ requires $r_{i j}<r_{\mathrm{c}}$, where $r_{i j}$ is the distance between $P_{i}$ and $P_{j}$. All the distances $r_{i j}$ consist of an array and its variance could be used to measure the degree of agglomeration. A larger "variance" corresponds to a more agglomerated system. $r_{\mathrm{c}}$ is chosen as $3 R_{\mathrm{p}}$ in the present paper so as to find a reasonable neighboring region for each particle.

In order to show intuitively the physical meaning of this parameter, simulation of a 2D system with 500 copper particles of radius $40 \mu \mathrm{m}$ is conducted under the condition of $1050^{\circ} \mathrm{C}$ sintering temperature with an inter-particle tangential viscosity of 0.01 . Agglomeration develops with sintering as shown in the insets of Fig. 2, in which the non-uniformity of the distribution of particles increases and leads to an increasing "variance".

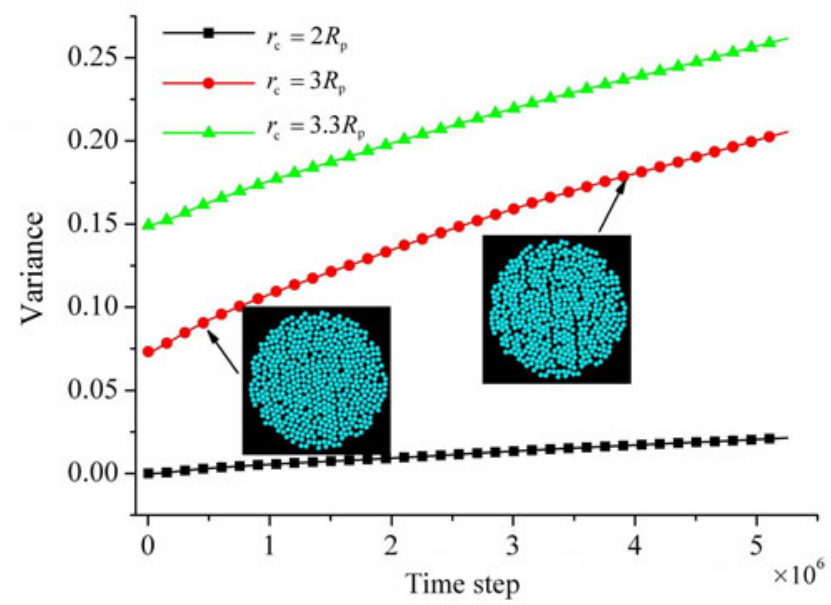

Fig. 2 The "variance" as a function of the time step for a 2D system with 500 copper particles of radius $40 \mu \mathrm{m}$ under sintering temperature $1050^{\circ} \mathrm{C}$ and inter-particle tangential viscosity 0.01

\section{Revision of the DEM in Olmos et al. [19]}

In this paper, the force law in Eq. (2) is the same as that used by Olmos et al. [19]. In Ref. [19] the movement of each particle consisted of two steps: the first one was an affine transformation of particles and the second one was a rearrangement process leading to mechanical equilibrium. As they stated, "The first step may be too strong and may limit further rearrangement." In our simulations, the first step in Olmos et al. [19] is completely given up and the movement of each particle is naturally produced by the interactions of contacting particles.

Figure 3 shows the average value of rearrangement parameter, i.e., the mean deviation angle $\bar{\theta}$ of all particles in the system, as a function of the densification $\left(D-D_{0}\right) / D_{0}$. The experimental curve labeled as "experiment by Olmos [19]" in Fig. 3 was obtained by Olmos et al. [19, 20] using in situ X-ray micro-tomography. In their experiments, a closepacked copper powder with radius in the range of $0-63 \mu \mathrm{m}$ was adopted with an initial relative density of 0.645 and the sintering temperature was $1050^{\circ} \mathrm{C}$. DEM simulations were also carried out by them, and the results are labeled as "DEM by Olmos [19]" in Fig. 3. "DEM-22", "DEM-30", "DEM40 " and "DEM-50" refer to the present simulations for particle radius of $22 \mu \mathrm{m}, 30 \mu \mathrm{m}, 40 \mu \mathrm{m}$ and $50 \mu \mathrm{m}$ with the same temperature $1050{ }^{\circ} \mathrm{C}$ and the same inter-particle tangential viscosity 0.001 as used in Ref. [19]. Experimental results show a very strong rearrangement of particles at the beginning of sintering and the mean deviation angle decreases continuously to a constant value $18^{\circ}$ with an increasing densification. Comparing the experimental results to both our simulation ones and those obtained by Olmos et al. [19] proves that our numerical method is more reasonable to predict the particle rearrangement than that in Olmos et al. [19].

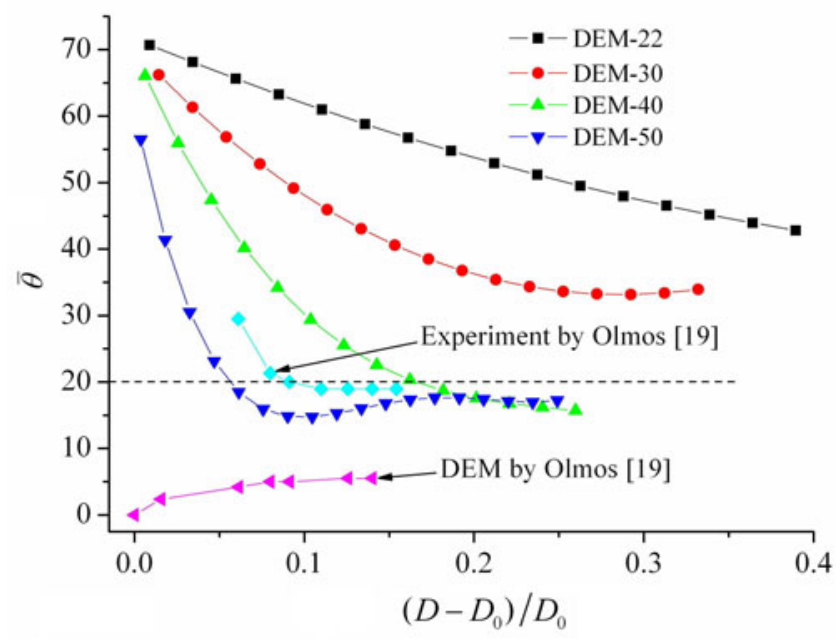

Fig. 3 The average value of the rearrangement parameter $\theta$ as a function of densification during copper particles sintering at temperature $1050^{\circ} \mathrm{C}$ 


\section{Numerical results and discussions}

The parameter "variance" proposed in Sect. 3.2 is used to characterize the degree of agglomeration in this sub-section. The effects of particle size, particle size distribution, temperature, initial volume fraction, initial distribution of particles and inter-particle viscosity on agglomeration are analyzed.

Figure 4 shows the effect of particle size on agglomeration, in which six kinds of cases are considered with the same initial distribution of particles but different particle sizes, i.e. $22 \mu \mathrm{m}, 30 \mu \mathrm{m}, 40 \mu \mathrm{m}, 50 \mu \mathrm{m}, 60 \mu \mathrm{m}$ and $70 \mu \mathrm{m}$. The sintering temperature is set to $1050{ }^{\circ} \mathrm{C}$ and a small tangential viscosity 0.001 is adopted. Because of the same initial distribution of particles, the values of the initial "variance" for the six cases are nearly identical. The "variance" is nonzero at the beginning of simulations due to the random packing which induces a slightly non-uniform distribution of particles. At a given densification, the system with relatively small particles has a high agglomeration degree. The "variance" increases with the densification of sintering systems and a maximum "variance" exists if the particle size is smaller than $50 \mu \mathrm{m}$. When the particle size is larger than $50 \mu \mathrm{m}$, the effect of particle size on the "variance" is very weak. With increasing densification, the system with large particles becomes more and more uniform in contrast to the initial state, which leads to a decreasing "variance". In a word, systems consisting of relatively smaller particles are more liable to agglomeration, which is consistent with the generally experimental finding.

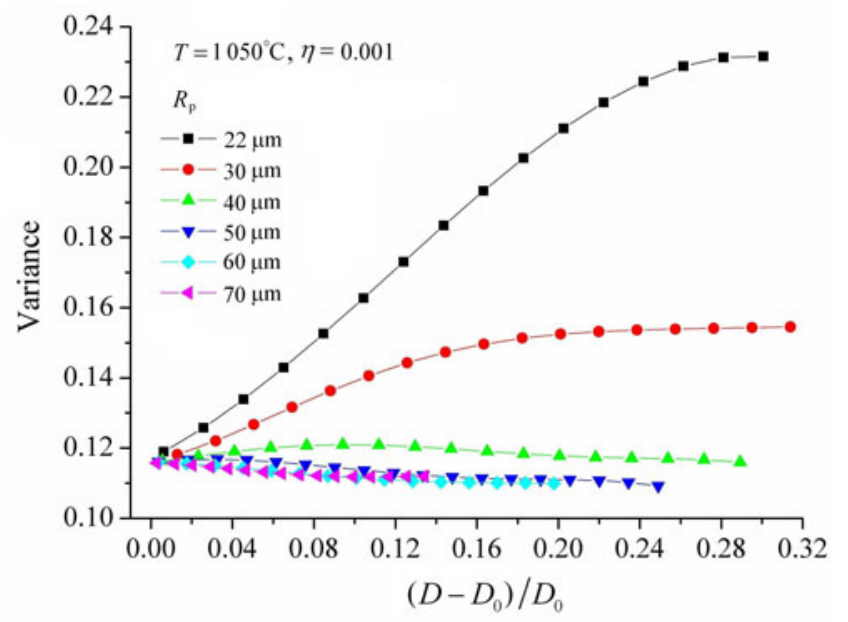

Fig. 4 The "variance" as a function of densification for systems with particle sizes of $22 \mu \mathrm{m}, 30 \mu \mathrm{m}, 40 \mu \mathrm{m}, 50 \mu \mathrm{m}, 60 \mu \mathrm{m}$ and $70 \mu \mathrm{m}$

Figure 5 shows the effect of viscosity on the agglomeration for a system with particles of radius $22 \mu \mathrm{m}$ and sintering temperature $1050{ }^{\circ} \mathrm{C}$. For a given densification, particles in the system with a larger inter-particle tangential viscosity distribute more uniformly, which corresponds to a smaller "variance". Since larger tangential viscosity parameter $\eta$ re- sults in a more difficult particle rearrangement, the particle agglomeration is also constrained. One should note that the forepart of some curves in Fig. 5 seems abnormal. This is because Fig. 5 shows the "variance" as a function of densification $\left(D-D_{0}\right) / D_{0}$, not a function of the sintering time. Figure 6 shows the "variance" as a function of time step. The initial values of "variance" of all curves in Fig. 6 are the same due to an identically initial distribution of particles. From Fig. 6, one can also see that a system with small inter-particle tangential viscosity tends to become more nonuniform compared with large tangential viscosity case. For cases with larger viscosity, "variance" will decrease with further densification as the sintering time goes on.

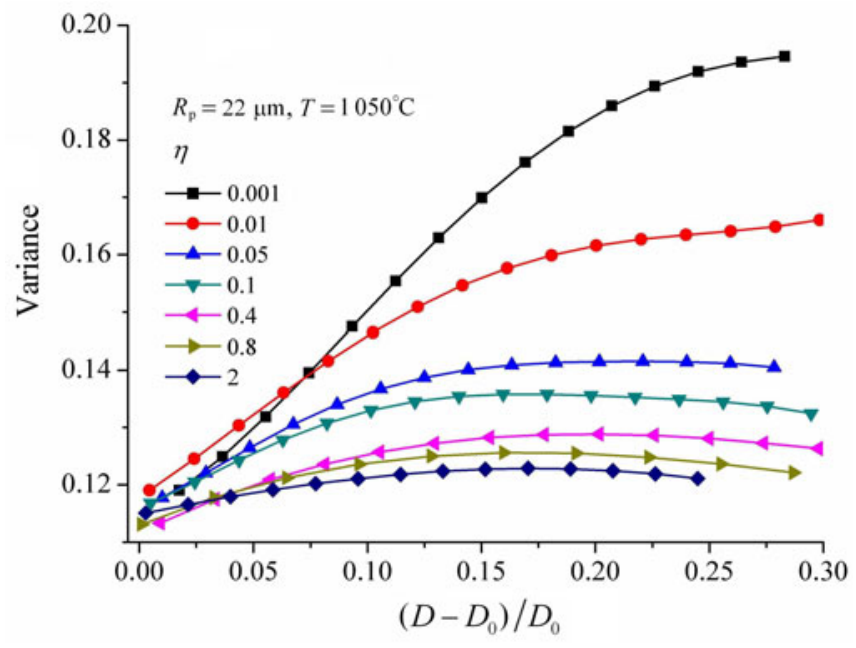

Fig. 5 The "variance" as a function of densification for systems with particle size $22 \mu \mathrm{m}$ and different inter-particle tangential viscosity $(0.001,0.01,0.05,0.1,0.4,0.8$ and 2$)$

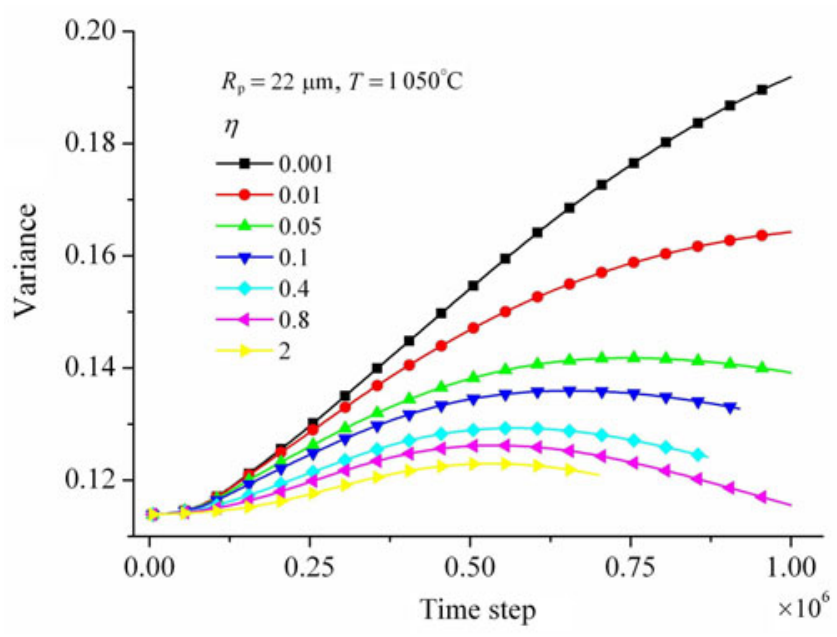

Fig. 6 The "variance" as a function of time step for systems with particle size $22 \mu \mathrm{m}$ and different inter-particle tangential viscosities $(0.001,0.01,0.05,0.1,0.4,0.8$ and 2$)$ 
Figure 7 shows the effect of viscosity $\eta$ on agglomeration for a system with particles of radius $50 \mu \mathrm{m}$ and temperature $1050^{\circ} \mathrm{C}$. The result for $\eta=0$ is also shown in Fig. 7 for comparison. From Fig. 7, one can see that viscosity inhibits the formation of agglomerates, which corresponds to a more uniform distribution of particles and a decreasing "variance". Comparing the results in Fig. 5 for a system with small particles and those in Fig. 7 for a system with large particles, it shows that the viscosity in the latter inhibits the formation of agglomerates more significantly than that in the former.

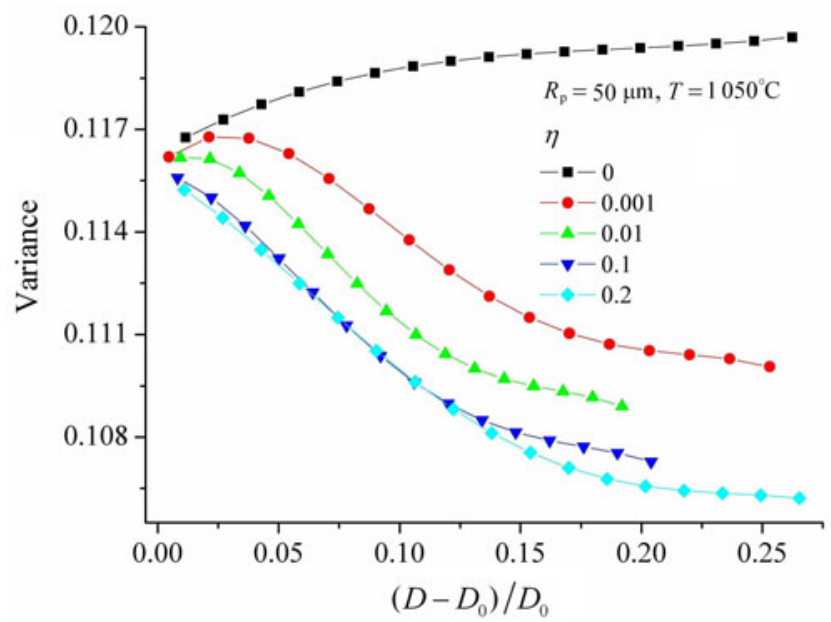

Fig. 7 The "variance" as a function of densification for systems with particle size $50 \mu \mathrm{m}$ and different inter-particle tangential viscosities $(0,0.001,0.01,0.1$ and 0.2$)$

Figure 8 shows the effect of viscosity $\eta$ on the densification of a system with particles of radius $22 \mu \mathrm{m}$ at sintering temperature $1050{ }^{\circ} \mathrm{C}$. It is surprising to find that at the same sintering time, the densification increases with the increase of viscosity from 0.01 to 0.2 , but decreases when the viscosity increases from 0.2 to 2 . This can be explained from the agglomeration point of view. Drastic particle rearrangement due to the adoption of a small viscosity induces strong agglomeration which is detrimental to the shrinkage of the whole system. Hence, a small viscosity can restrain the particle agglomeration and enhance the densification of the system. But a large tangential viscosity strongly restrains the movement of particles and finally induces retardation of the shrinkage of the system. This result shows that particle rearrangement is not always beneficial for the densification of the whole system, because strong particle rearrangements would induce drastic particle agglomerations which lead to the occurrence of large pores between different agglomerates. This is different from the result of Henrich et al. [26], in which they found that the densification rate was greatly enhanced by particle rearrangement with a small viscosity. In their simulations, two artificial techniques, i.e. a small isostatic pressure and an extended interaction radius, are used to initiate the rearrangement when they find the particles do not feel open gaps. In our opinion, the formation of open gaps is a physical process and it is inappropriate to annihilate them using any non-physical method. The formation of open gaps could well be attributed to strong agglomeration. One possible reason for the annihilation of open gaps is the sintering of agglomerates which is not the focus of this paper and the movement of agglomerates is not included in the present simulations.

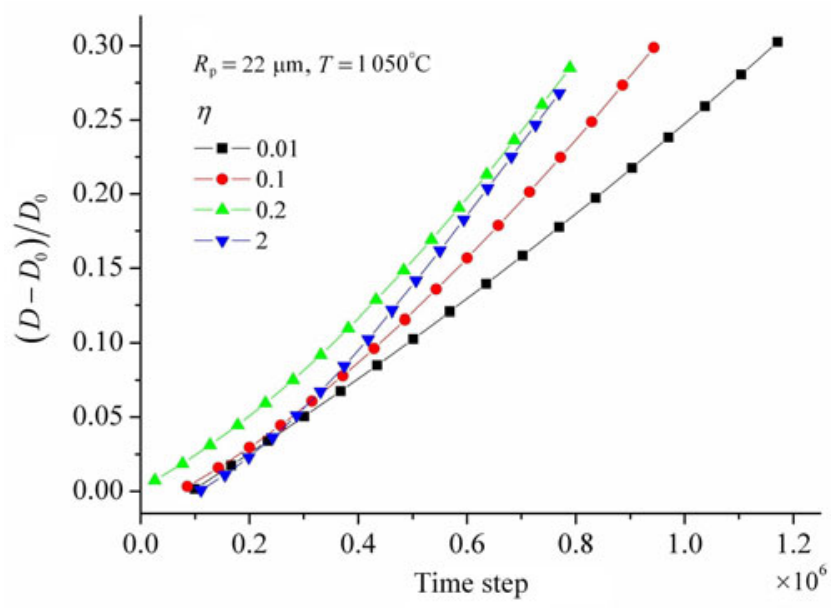

Fig. 8 The densification as a function of the time step for systems with particle radius $22 \mu \mathrm{m}$ and different inter-particle tangential viscosities (0.01, 0.1, 0.2 and 2)

Temperature is an easy-controlling parameter in sintering experiments. Figure 9 shows the influence of temperature on the agglomeration of a system with particles of radius $22 \mu \mathrm{m}$, in which the tangential viscosity is taken as 0.001 . With a fixed densification, the system under higher temperature will exhibit more agglomerates, which corresponds to a large value of "variance". This is because particles would rearrange more drastically under a higher temperature condition as shown in Fig. 10. Figure 10 shows the average value

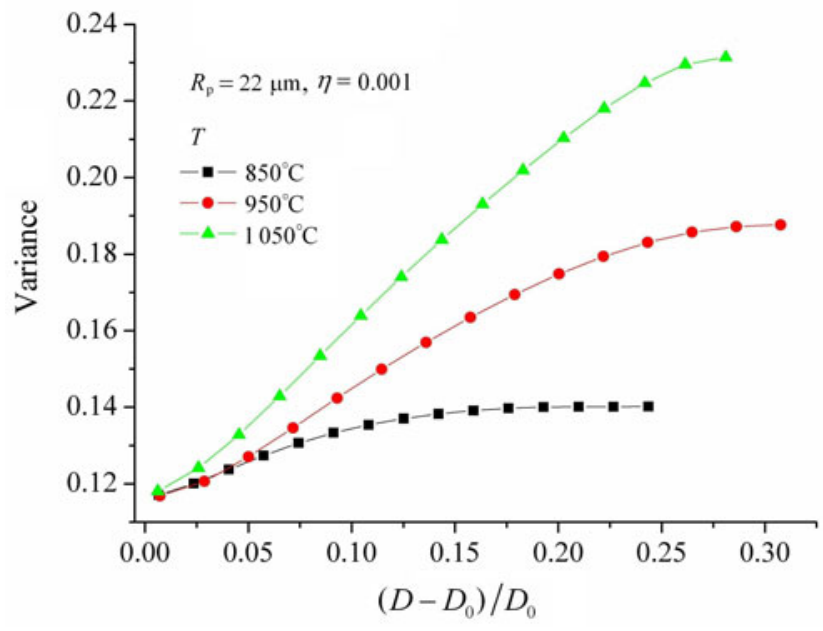

Fig. 9 The "variance" as a function of densification for systems with particle radius $22 \mu \mathrm{m}$, tangential viscosity 0.001 and sintering temperature of $850^{\circ} \mathrm{C}, 950^{\circ} \mathrm{C}$ and $1050^{\circ} \mathrm{C}$ 


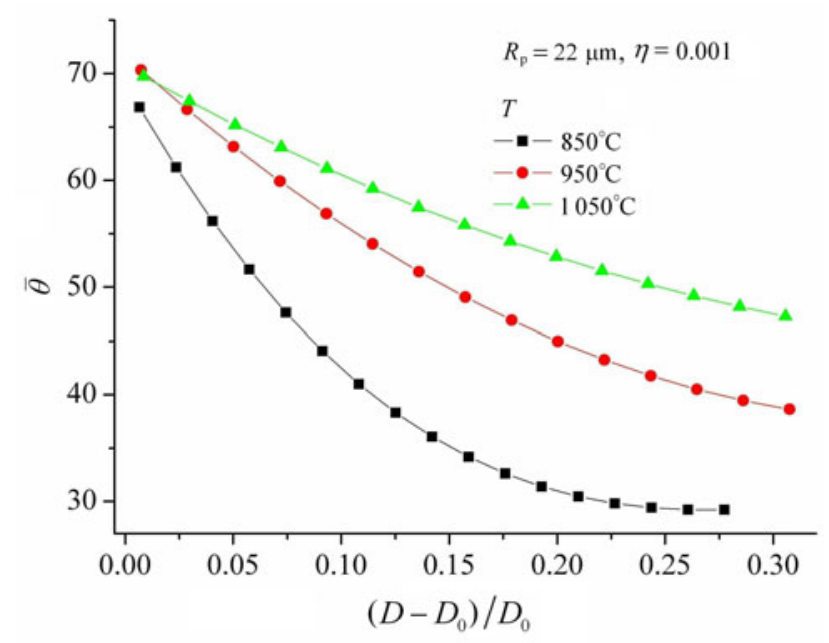

Fig. 10 The average value of rearrangement parameter $\theta$ as a function of densification for systems at sintering temperatures of $850^{\circ} \mathrm{C}$, $950^{\circ} \mathrm{C}$ and $1050^{\circ} \mathrm{C}$

of rearrangement parameter $\theta$ as a function of densification. For a given densification, $\bar{\theta}$ increases with increasing sintering temperature, which means the degree of agglomeration can be adjusted by tuning the sintering temperature.

The effect of initial distribution of particles on agglomeration is shown in Fig. 11. Three samples produced by the random packing are considered with the same particle size of $40 \mu \mathrm{m}$, the tangential viscosity of 0.001 , the temperature of $1050{ }^{\circ} \mathrm{C}$ and the initial volume fraction of $62 \%$. The initial values of "variance" are different, which means different distributions of particles caused by the random packing. As densification increases, the "variance" of each system takes different value but with a similar variation tendence. It means that the initial distributions of particles show significant effect on the agglomeration but without changing the tendency of the results.

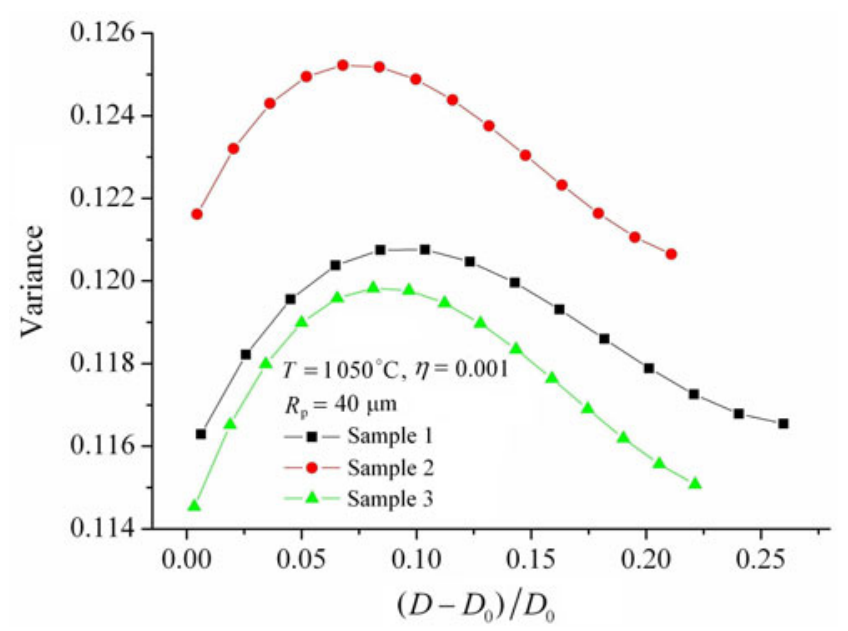

Fig. 11 The "variance" as a function of densification for systems with different initial distributions of particles
Figure 12 shows the effect of particle size distribution on agglomeration, in which the second model mentioned in Sect. 3 is adopted. Simulations are conducted under $1050^{\circ} \mathrm{C}$ and the inter-particle tangential viscosity is taken as 0.4 . The mean size of particles is $22 \mu \mathrm{m}$ with three different standard deviations, 0.02, 0.06 and 0.08. For each case, three samples produced by the random packing are investigated and the results shown in Fig. 12 for each case are average ones. From Fig. 12, one can see that a system with a large standard deviation of particle size will have a large "variance" for a given densification. In other words, the broader the distribution of particle size, the stronger the agglomeration.

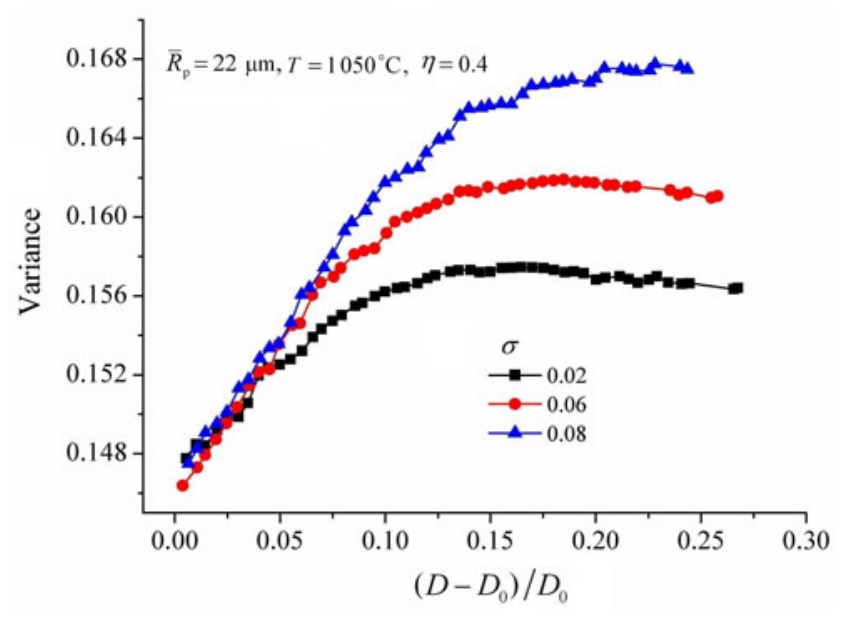

Fig. 12 The "variance" as a function of densification for systems with different standard deviations of particle size distributions

The third model mentioned in Sect. 3 is used to investigate the effect of initial volume fraction on agglomeration. The simulation system consists of uniform particles with radius of $22 \mu \mathrm{m}$. The sintering temperature is $1050{ }^{\circ} \mathrm{C}$ and the inter-particle tangential viscosity is 0.2 . Figure 13 shows the "variance" as a function of the densification for different cases with volume fractions of $50 \%, 52 \%, 56 \%$ and $60 \%$. The initial average coordination numbers for the systems with the above different volume fractions are about $3.50,3.70,4.12$ and 4.54, respectively. In order to eliminate the influence of the distribution of particles, average results are adopted from three samples for each case as shown in Fig. 13. The initial value of "variance" for the system with $50 \%$ volume fraction is a little larger than that of the other three cases with a larger volume fraction, which is due to the more non-uniform distribution of particles in the system with a small volume fraction. For a given densification, the system with a small initial volume fraction will exhibit stronger agglomeration.

As a whole, six factors have been studied in the present work, which can be divided into two categories. The first group consists of particle size, particle size distribution and 


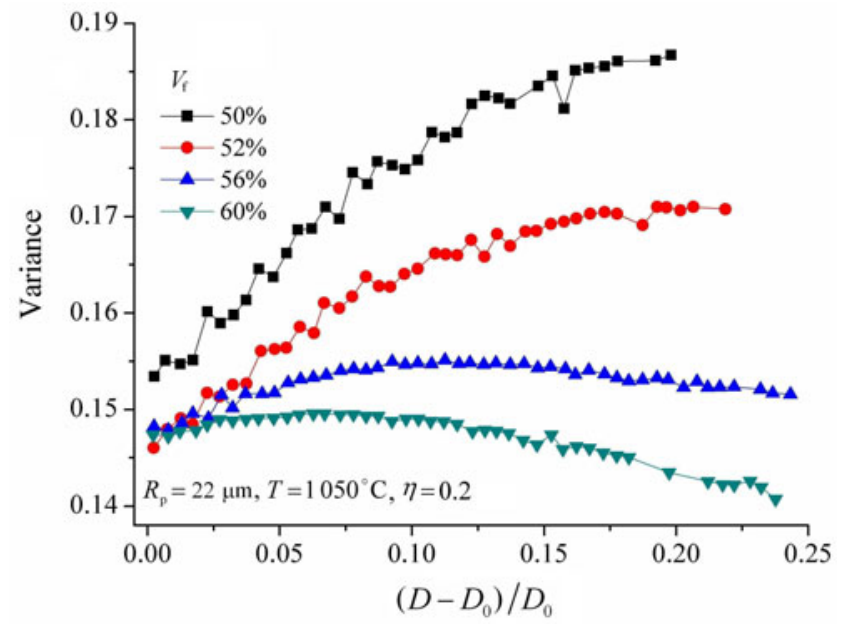

Fig. 13 The "variance" as a function of densification for systems with different initial volume fractions

temperature, which affect the sintering rate of two contacting particles through the force law given in Eq. (2). It should be noted that if the radii of two contacting particles are different, the equivalent radius $R^{*}$ should be used. Another group includes inter-particle viscosity, initial volume fraction and initial distribution of particles, which determine the regulating ability of particles in a system. The degree of agglomeration of a system is determined by two factors: the sintering rate of two contacting particles and the regulating ability of the movement of particles. When the sintering rate is high while the regulating ability is weak, agglomeration happens. This opinion is consistent with that suggested by Kadusnikov et al. $[14,15]$.

\section{Conclusions}

Particle agglomeration is widely observed in sintering experiments. Because of some realistic difficulties in experimental and theoretical researches, few works on particle agglomeration have been done to explore its effect on sintering, but its influence on the microstructural evolution and macroscopic properties of the sintered products is important. In this paper, we propose a new parameter "variance" to denote the change of distances between neighboring particles, which is used to describe the degree of agglomeration in a three-dimensional solid-state sintering system. The effects of a series of factors on agglomeration are investigated, including particle size, size distribution, inter-particle tangential viscosity, temperature, initial distribution of particles and initial volume fraction. It is found that when the other factors are given, agglomeration is apt to occur in systems with smaller particles, broader particle size distribution, higher temperature, smaller tangential viscosity or smaller initial volume fraction. Different initial distributions of particles induce different agglomerations. This study should be useful for understanding the role of agglomeration and the micro-structural evolution during sintering, as well as the design of sintering products with specific mechanical properties.

Acknowledgements We would like to thank Mr. Lei Chen for his constructive suggestion on the description of agglomeration and Mr. Cun Zhang for his helpful discussion on the numerical program.

\section{References}

1 Reeve, K.: Non-uniform shrinkage in sintering. Am. Ceram. Soc. Bull. 42, 452 (1963)

2 Petzow, G., Exner, H.E.: Particle rearrangement in solid-state sintering. Z. Metallk. 67, 611-618 (1976)

3 Exner, H.E., Petzow, G.: Shrinkage and rearrangement during sintering of glass spheres. In: Kuczynski, G.C. ed. Sintering and Catalysis. Plenum Press Publ. Corp., New York 279-293 (1975)

4 Weiser, M.W., Dejonghe, L.C.: Rearrangement during sintering in two-dimensional arrays. J. Am. Ceram. Soc. 69, 822826 (1986)

5 Claussen, N., Exner, H.E.: Influence of sintering on fine powder compacts for catalyst application. Powder Metall. 15, 202215 (1972)

6 Ada, K., Onal, M., Sarikaya, Y.: Investigation of the intraparticle sintering kinetics of a mainly agglomerated alumina powder by using surface area reduction. Powder Technol. 168, 37-41 (2006)

7 Kuo, J., Bourell, D.L.: Structural evolution during calcination of sol-gel synthesized alumina and alumina- 8 vol\% zirconia composite. J. Mater. Sci. 32, 2687-2692 (1997)

8 Palmero, P., Lombardi, M., Montanaro, L., et al.: Effect of heating rate on phase and microstructural evolution during pressureless sintering of a nanostructured transition alumina. Int. J. Appl. Ceram. Technol. 6, 420-430 (2009)

9 Huppmann, W.J., Riegger, H.: Modeling of rearrangement processes in liquid-phase sintering. Acta Metall. 23, 965-971 (1975)

10 Linardos, S., Zhang, Q., Alcock, J.R.: An investigation of the parameters effecting the agglomerate size of a pzt ceramic powder prepared with a sol-gel technique. J. Eur. Ceram. Soc. 27, 231-235 (2007)

11 Trunec, M., Dobsak, P., Cihlar, J.: Effect of powder treatment on injection moulded zirconia ceramics. J. Eur. Ceram. Soc. 20, 859-866 (2000)

12 Rhodes, W.H.: Agglomerate and particle-size effects on sintering yttria-stabilized zirconia. J. Am. Ceram. Soc. 64, 19-22 (1981)

13 Lange, F.F.: Processing-related fracture origins. 1. Observations in sintered and isostatically hot-pressed $\mathrm{Al}_{2} \mathrm{O}_{3} / \mathrm{ZrO}_{2}$ composites. J. Am. Ceram. Soc. 66, 396-398 (1983)

14 Kadushnikov, R.M., Skorokhod, V.V.: Simulating zonal segregation in powder sintering. Sov. Powder Metall. Met. Ceram. 30, 557-561 (1991)

15 Kadushnikov, R.M., Alievskii, D.M., Alievskii, V.M., et al.: Computer-simulation for microstructure evolution in a polydisperse material on sintering. 2. Zoned segregation. Sov. Powder Metall. Met. Ceram. 30, 356-360 (1991) 
16 Martin, C.L., Bouvard, D., Delette, G.: Discrete element simulations of the compaction of aggregated ceramic powders. J. Am. Ceram. Soc. 89, 3379-3387 (2006)

17 Kim, J.C., Martin, D.M., Lim, C.S.: Effect of rearrangement on simulated particle packing. Powder Technol. 126, 211-216 (2002)

18 Ciftcioglu, M., Akinc, M., Burkhart, L.: Effect of agglomerate strength on sintered density for yttria powders containing agglomerates of monosize spheres. J. Am. Ceram. Soc. 70, C329-C334 (1987)

19 Olmos, L., Martin, C.L., Bouvard, D., et al.: Investigation of the sintering of heterogeneous powder systems by synchrotron microtomography and discrete element simulation. J. Am. Ceram. Soc. 92, 1492-1499 (2009)

20 Olmos, L., Takahashi, T., Bouvard, D., et al.: Analysing the sintering of heterogeneous powder structures by in situ microtomography. Philos. Mag. 89, 2949-2965 (2009)

21 Bernard, D., Gendron, D., Heintz, J.M., et al.: First direct $3 \mathrm{~d}$ visualisation of microstructural evolutions during sintering through x-ray computed microtomoaraphy. Acta Mater. 53, 121-128 (2005)

22 Lame, O., Bellet, D., Di Michiel, M., et al.: Bulk observation of metal powder sintering by $\mathrm{x}$-ray synchrotron microtomography. Acta Mater. 52, 977-984 (2004)

23 Vagnon, A., Riviere, J.P., Missiaen, J.M., et al.: 3d statistical analysis of a copper powder sintering observed in situ by synchrotron microtomography. Acta Mater. 56, 1084-1093 (2008)

24 Martin, C.L., Schneider, L.C.R., Olmos, L., et al.: Discrete element modeling of metallic powder sintering. Scr. Mater. 55, 425-428 (2006)

25 Martin, C.L., Bouvard, D., Shima, S.: Study of particle rearrangement during powder compaction by the discrete element method. J. Mech. Phys. Solids 51, 667-693 (2003)

26 Henrich, B., Wonisch, A., Kraft, T., et al.: Simulations of the influence of rearrangement during sintering. Acta Mater. 55, 753-762 (2007)

27 Wang, D., Zhou, Y.: Particle dynamics in dense shear granular flow. Acta Mech. Sin. 26, 91-100 (2010)
28 Olmos, L., Martin, C.L., Bouvard, D.: Sintering of mixtures of powders: Experiments and modelling. Powder Technol. 190, 134-140 (2009)

29 Parhami, F., McMeeking, R.M.: A network model for initial stage sintering. Mech. Mater. 27, 111-124 (1998)

30 Wonisch, A., Guillon, O., Kraft, T., et al.: Stress-induced anisotropy of sintering alumina: Discrete element modelling and experiments. Acta Mater. 55, 5187-5199 (2007)

31 Martin, C.L., Bordia, R.K.: The effect of a substrate on the sintering of constrained films. Acta Mater. 57, 549-558 (2009)

32 Martin, C.L., Camacho-Montes, H., Olmos, L., et al.: Evolution of defects during sintering: Discrete element simulations. J. Am. Ceram. Soc. 92, 1435-1441 (2009)

33 Cundall, P.A., Strack, O.D.L.: Discrete numerical-model for granular assemblies. Geotechnique 29, 47-65 (1979)

34 Thornton, C., Antony, S.: Quasi-static deformation of particulate media. Philos. Trans. R. Soc. A-Math. Phys. Eng. Sci. 356, 2763-2782 (1998)

35 Bouvard, D., McMeeking, R.M.: Deformation of interparticle necks by diffusion-controlled creep. J. Am. Ceram. Soc. 79, 666-672 (1996)

36 Wonisch, A., Kraft, T., Moseler, M., et al.: Effect of different particle size distributions on solid-state sintering: A microscopic simulation approach. J. Am. Ceram. Soc. 92, 14281434 (2009)

37 Wonisch, A., Kraft, T., Moseler, M., et al.: Discrete element simulations of constrained ceramic powder sintering. Ceramic forum international: CFI. Berichte der Deutschen Keramischen Gesellschaft 85, 18-23 (2008)

38 Parhami, F., McMeeking, R.M., Cocks, A.C.F., et al.: A model for the sintering and coarsening of rows of spherical particles. Mech. Mater. 31, 43-61 (1999)

39 Pan, J., Le, H., Kucherenko, S., et al.: A model for the sintering of spherical particles of different sizes by solid state diffusion. Acta Mater. 46, 4671-4690 (1998)

40 Fang, T.T., Hsieh, H.L.: Experimental assessment of modified statistical-theory of sintering. J. Mater. Sci. 27, 4639-4646 (1992) 